\title{
Estimation of In Vivo Water Content of the Stratum Corneum from Electrical Measurements
}

\author{
Gorm Krogh Johnsen*,1, Ø.G. Martinsen ${ }^{1,2}$ and S. Grimnes ${ }^{1,2}$ \\ ${ }^{I}$ Department of Physics, University of Oslo, P.O. Box 1048 Blindern, N-0316 Oslo, Norway \\ ${ }^{2}$ Department of Clinical and Biomedical Engineering, Rikshospitalet, Oslo University Hospital, N-0027 Oslo, Norway
}

\begin{abstract}
In vivo water content in the epidermal stratum corneum can be estimated by means of low frequency susceptance measurements. In the in vitro calibration necessary to find the in vivo water content, the stratum corneum will have a uniform distribution of water across its thickness. However, in vivo stratum corneum has an increasing water concentration profile from the outermost towards the innermost parts. This paper will investigate the possibility of estimating the equilibrium water content in the in vivo stratum corneum non-invasively from electrical susceptance measurements. Given a known shape of the water concentration profile in the in vivo stratum corneum and the dependence of susceptance on the water content, it is possible to calculate the water content in vivo based on analytically derived expressions for the water concentration profile. A correspondence between in vivo and in vitro water content needed for this purpose is also established.
\end{abstract}

Keywords: Bioimpedance, electrical susceptance, stratum corneum, hydration.

\section{INTRODUCTION}

The vital functions of the stratum corneum (S.C.) are strongly dependent on the water content therein [1-3]. Knowledge of the water content of the S.C. in vivo has been shown to be an important factor in clinical determinations of non-visible skin diseases such as atopic eczema [4-7]. Electrical methods for measuring skin hydration have been studied for several decades and a low frequency susceptance is found to be the most adequate [8-10]. In this paper the term low frequency means a constant frequency below 100 $\mathrm{Hz}$. In general higher frequencies will measure also on deeper skin layers [11]. Furthermore, the conductance of the S.C. is influenced by sweat duct filling which is an unwanted error to the measurements. The method of Martinsen et al. [12] is not affected by sweat duct filling, viable skin or temperature as it is focused on S.C. only [9]. Often a fourelectrode system is used for the electrical bioimpedance measurements [13], but the susceptance data used in this paper are found with monopolar recording using a threeelectrode setup following the principles of Grimnes [14].

In this theoretical paper we want to investigate the possibility of estimating the equilibrium water content of the in vivo S.C. by electrical susceptance measurements. The method we propose in this paper is non-invasive, rapid and simple. The estimation of the in vivo S.C. water content is done by calculating the relation between in vivo and in vitro susceptance, and thereby also the connection between the corresponding water contents. Viable skin contains about 70$80 \%$ water [1], whereas the surface of the S.C. is drier. There will therefore be a net water gradient throughout the

*Address correspondence to this author at the Department of Physics,University of Oslo, P.O. Box 1048 Blindern, NO-0316 Oslo, Norway; Tel: +4722854077; Fax: +4722856422; E-mail: gormj@ fys.uio.no
S.C. In vitro, however, the water content may be kept uniform, due to a moisture chamber as in the measurements of Martinsen et al. [12], where an exponential correspondence is found between measured susceptance and water content of the S.C. given in gram water per gram dry skin. This means that any in vivo susceptance corresponds to an in vitro water content. As also discussed in Johnsen et al. [15], assuming a linear water concentration profile in the in vivo S.C. it is possible to analytically find a relation between in vitro and in vivo measurements, and therefore also possible to predict the water concentration profile which yields the water content of in vivo S.C. The linearity of the water concentration profile is indicated in the literature [16-20]. Also from diffusion theory the water concentration profile of the S.C. will be linear as a first approximation. The passive diffusion of water across the S.C. is governed by Fick's laws of diffusion. For the equilibrium transport of water across the thickness of the S.C. Fick's law [21, 22] yields

$$
F=D \frac{d C(z)}{d z},
$$

where $F$ is the flux of water, $D$ is the diffusion constant, and $C(z)$ is the concentration of water across the S.C. thickness, $z$. A constant value of $D$ gives a linear gradient of water in the S.C. whereas a non-constant value, as indicated by [20, 23], will give deviations from linearity. However, a slight variation in the value of $D$ with water content results in only small deviations from linearity with respect to the water concentration profile in the S.C. $[20,22]$ where the former predicts linear water profiles based on a non-constant value of $D$ with respect to the water content of the S.C. We will show that the assumption that the concentration profile being linear is valid for our calculations even if the profile deviates from linearity, i.e. containing also higher order terms. Thus, as a first approximation we consider the in vivo water concentration profile to be linear across the entire S.C. when 
we perform our estimates of in vivo water content. Any given analytic expression of the water profile will in turn make it possible to estimate in vivo water content.

\section{METHODS}

The S.C. is modeled so that the water content increases with relative depth, $z$, where $z \in[0,1]$. The S.C. consists of dead and deformed keratinised cells containing water in and around them [24]. This results in strong capacitive properties at low frequencies $[25,26]$, and a section across the S.C. thickness is therefore modeled as a series of capacitors, each with infinitesimal thickness and a dielectric dependency on the water content at the given depth. Thus, the S.C. is viewed as a continuous series of capacitors and resistors as illustrated in Fig. (1). The current density will be equal in all infinitesimal slices of the S.C. that is being considered (i.e. under the electrode). This is ensured by using a threeelectrode setup as described first by Grimnes [14]. For sufficiently low frequencies, that is, below $100 \mathrm{~Hz}$, impedivity of the S.C. is much higher than for viable tissue [27]. In addition, the much larger dimension of the measuring electrode compared to the S.C. thickness also justifies a uniform representation of the current density across the S.C. thickness. Yamamoto and Yamamoto [28] report on an upper limit of linearity of skin current density to be about $10 \mu \mathrm{A} / \mathrm{cm}^{2}$ at $10 \mathrm{~Hz}$ and $100 \mu \mathrm{A} / \mathrm{cm}^{2}$ at $100 \mathrm{~Hz}$. In this paper we will be operating well within the linear range. Electrical admittance is basically measured by applying a constant voltage and measuring the corresponding current. Furthermore, a lock-in amplifier is used to measure only the imaginary part of the admittance, i.e. the susceptance. As a general model of the water concentration profile we will introduce a linear term and also higher order terms, covering a broad class of profile shapes (depending on the values of the parameters)

$w=a+b z+c z^{2}$,

where $w$ is water content given in gram water per gram wet skin (i.e. skin and water), and $a$ (dimensionless), b

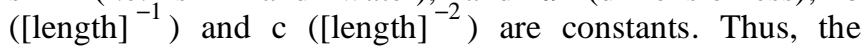
outermost water content of the S.C. is then given by $a$ and

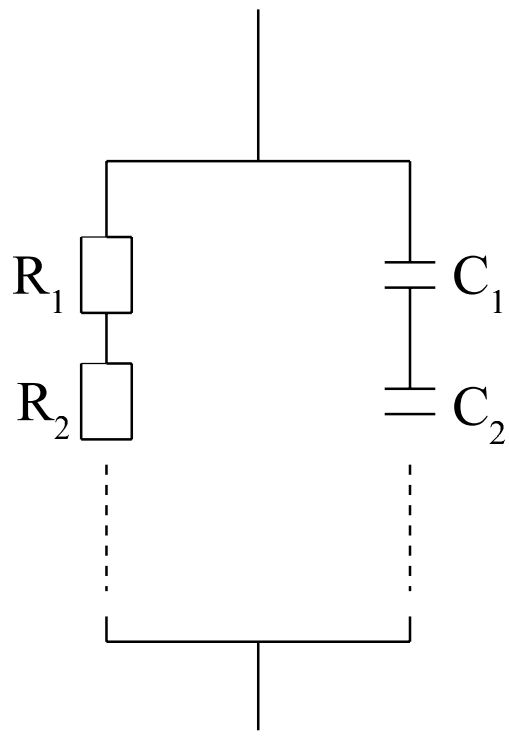

Fig. (1). The electrical model of the stratum corneum. the innermost by $d=a+b+c$. The parameters of the water concentration profile in our model are unbiased, meaning that the value of $b$ and $c$ can be negative giving a negative slope. Such a shape of the profile may appear if the relative humidity of the ambient air is sufficiently high giving a larger value of $a$ than $d$.

The exponential behavior of the susceptance with respect to in vitro water content, $v$ given in gram water per gram dry skin found in Martinsen et al. [12], then yields the general form of the susceptance

$B=B_{0} \exp (\alpha v)$

where $B_{0}$ represents the susceptance measured when the S.C. is only one, uniform dielectric, and $\alpha$ is an experimental constant that can be found in Martinsen et al. [12] in the case of in vitro S.C. taken from the heel. We want to use the linearity of the water concentration profile in the S.C. to find in vivo water content. However, the susceptance depends exponentially on gram water per gram dry skin and must therefore be converted to be a function of gram water per gram wet skin, that is, a function of $w$ rather than $v$, in order to be combined with the linear water distribution. Converting $v$ into $w$ is done by

$w=\frac{v \rho_{s} \rho_{w}}{v \rho_{s}+\rho_{w}}$,

where $\rho_{s}$ and $\rho_{w}$ are the densities of dry S.C. and water, respectively. The density of S.C. is estimated by Scheuplein [29], and the density of water is set to $1.00 \mathrm{~g} \mathrm{~cm}^{-3}$. Eq. (3) then turns into

$B=B_{0} \exp \left(\frac{\alpha}{\rho_{s}} \frac{a+b z+c z^{2}}{1-a-b z-c z^{2}}\right)$

An expression of the infinitesimal in vivo impedance, $\mathrm{d} Z$, for each thin capacitor in our model can be found through the relation $X=(\omega C)^{-1}$, where $X$ is reactance, $\omega$ is signal frequency, at which the susceptance is being measured, and $C$ is the capacitance depending on the water content of the dielectric. It is worth noticing that the outermost and driest parts of the S.C. in general will contribute more to the impedance than the deeper layers. Using that relative permittivity is proportional to susceptance, we get that

$$
\begin{aligned}
& \frac{d Z}{d z}=\frac{1}{\omega A \varepsilon_{0} \varepsilon(w)} \\
& =\frac{1}{\omega A \varepsilon_{0}} \exp \left(-\frac{\alpha}{\rho_{s}} \frac{a+b z+c z^{2}}{1-a-b z-c z^{2}}\right),
\end{aligned}
$$

where $A$ is the cross-section area of the S.C. region being considered, $\varepsilon_{0}$ is the electrical permittivity of vacuum, and $\varepsilon(w)$ is the relative dielectric permittivity which is proportional to the susceptance and therefore also a function of the water content, $w$. Total in vivo impedance of the S.C. is found by integrating up (7) 


$$
Z=\frac{1}{\omega A \varepsilon_{0}} \int_{0}^{1} \exp \left(-\frac{\alpha}{\rho_{s}} \frac{a+b z+c z^{2}}{1-a-b z-c z^{2}}\right) d z
$$

We would now like to find the in vitro water content, $w_{\text {in_vitro }}$ that corresponds to the in vivo impedance given in (8). This is done by combining the in vivo result with the fact that impedance decreases exponentially with the water content, w (gram water per gram dry skin). Thus, from (8) we get that

$Z=\frac{1}{\omega A \varepsilon_{0}} \exp \left(-\alpha v_{\text {in }- \text { vitro }}\right)$,

which results in

$v_{\text {in- vitro }}=-\frac{1}{\alpha} \ln \left[\int_{0}^{1} \exp \left(-\frac{\alpha}{\rho_{s}} \frac{a+b z+c z^{2}}{1-a-b z-c z^{2}}\right) d z\right]$

The in vitro water content, $w$ ( gram water - gram wet tissue ) is then given by

$$
w_{\text {in_vitro }}=\frac{v_{\text {in_vitro }} \rho_{s}}{v_{\text {in_vitro }} \rho_{s}+1},
$$

with $v_{\text {in_vitro }}$ given as in (10).

\section{RESULTS}

The correspondence between in vivo and in vitro water content is given by the set of equations (10) and (11) where the in vivo water content is given by the right-hand-side (RHS) of (10). We will now show that the estimated value of in vitro water content, based on any physical in vivo concentration profile is more or less the same when $c=0$ (linear profile) as when $\mathrm{c} \neq 0$ (deviations from linearity). For any measured in vivo susceptance value, the water concentration profiles must satisfy the condition

$f\left(v_{\text {in_vitro }}, a, b, c\right)=0$,

where

$f\left(v_{\text {in_vitro }_{1}}, a, b, c\right)=v_{\text {in_vitro }}+$

$\frac{1}{\alpha} \ln \frac{1}{\omega A \varepsilon_{0}} \int_{0}^{1} \exp \left(-\frac{\alpha}{\rho_{s}} \frac{a+b z+c z^{2}}{1-a-b z-c z^{2}}\right) d z$

We see that the water concentration profiles are in general not unique for a given value of the in vitro water content (i.e. for an in vivo susceptance) due to the fact that both $a$ and $c$ are unknowns. This is as expected since two independent parameters can not be obtained from a single measurement. Hence, an in vivo susceptance will in general not be sufficient to determine the profile of the water concentration of the S.C. exactly, but rather serve us with a continuum of possible solutions.

The assumption that in vivo water content of the S.C. can be estimated from susceptance measurements and a linear concentration profile is reasonable as a first approximation. We show this in Tables $\mathbf{1}$ and $\mathbf{2}$, and Fig. (2) where we compare the results of in vitro estimated values for a purely linear concentration profile (i.e. $c=0$ ) and profiles containing also non-linearities for two different cases. The
Table 1. In Vitro Water Contents Corresponding to a Linear Water Concentration Profile and Non-Linear Profiles when $a=0.212$ and $b=0.288$. The Constants $a$, $b$, and $c$ are as defined in Eq. (2)

\begin{tabular}{|c|c|}
\hline $\mathbf{c}$ & $\mathbf{v}_{\text {in_vitro }}$ \\
\hline \hline$-0,4$ & 9 \\
\hline$-0,25$ & 16,3 \\
\hline$-0,1$ & 19,32 \\
\hline 0 & 20 \\
\hline 0,1 & 20,42 \\
\hline 0,25 & 20,86 \\
\hline 0,25 & 21,39 \\
\hline
\end{tabular}

Table 2. In Vitro Water Contents Corresponding to a Linear Water Concentration Profile and Non-Linear Profiles when $a=0.132$ and $b=0.268$. The Constants $a$, $b$, and $c$ are as defined in Eq. (2)

\begin{tabular}{|c|c|}
\hline $\mathbf{c}$ & $\mathbf{v}_{\text {in_vitro }}$ \\
\hline \hline$-0,4$ & 11,4 \\
\hline$-0,25$ & 27,12 \\
\hline$-0,1$ & 29,53 \\
\hline 0 & 30 \\
\hline 0,1 & 30,3 \\
\hline 0,25 & 30,67 \\
\hline 0,25 & 31,1 \\
\hline
\end{tabular}

deviation is significant only for very large negative (or positive) values of $c$. Physically, the numeric value of the parameter $c$, governing the deviation from linearity, may be limited to the interval $[-0.5,0.5]$. This is due to the fact that one can not have negative values of the S.C. water content. Values exceeding the relative value of $100 \%$ are also unphysical. The most likely value of $c$ is that it is slightly positive due to the fact that there is a gradient from the outermost S.C. (dry) towards the interface at the vial epidermis (wet). We note that the deviations from the linear results are small in this manner. Thus, it seems to be a reasonable approximation to model the concentration profile to be linear when we want to find the correspondence between in vivo and in vitro water content, and the term $c z^{2}$ will therefore be omitted in the following. Water content of the S.C. in vivo can possibly be found on the basis of the water concentration profiles. The profiles are in general given by the RHS of (10). We can think of this process of estimating in vivo water content as illustrated schematically in Fig. (3). Any susceptance corresponds to an in vitro water content which again is equivalent with in vivo water content in the S.C. It should be commented that whereas the relation between in vitro susceptance and water content in Fig. (3) is one-to-one, the correspondence between in vitro water 
content and in vivo water content is not unique. This is due to the fact that $\mathrm{a}, \mathrm{b}$ and $\mathrm{c}$ are in general not constant.

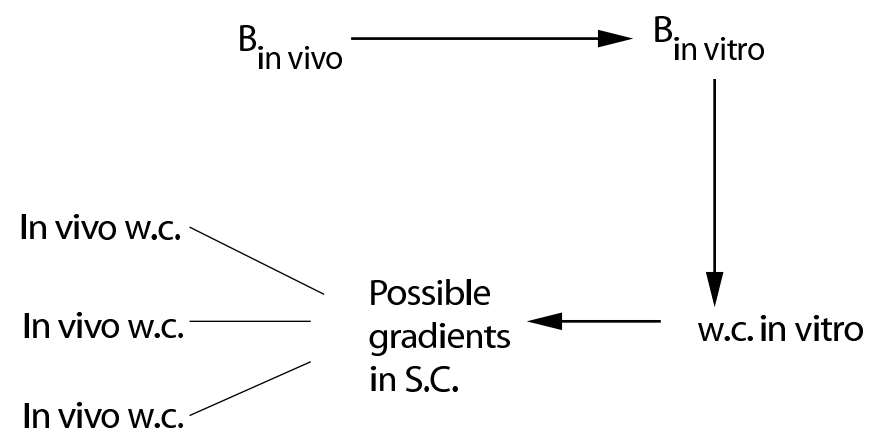

Fig. (2). The derivation of possible in vivo water gradients and then again in vivo water content with basis in an in vivo susceptance measurement.

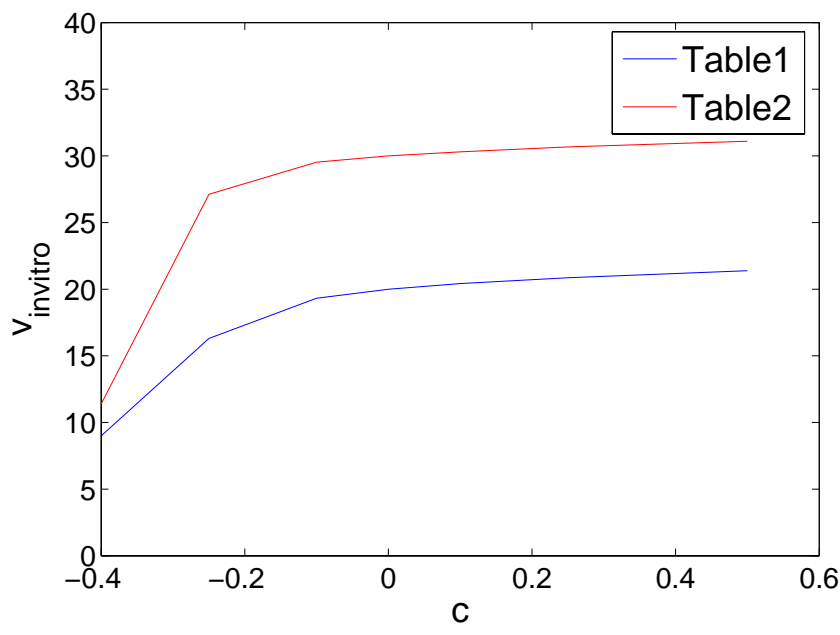

Fig. (3). The data in Table 1 and 2 illustrated graphically. Note the nearly flat curves for positive and slightly negative values of $c$.

\section{DISCUSSION}

We have in this paper studied the possibility of estimating the water content in the S.C. by means of electrical susceptance measurements performed in vivo. We have found a correspondence between in vivo and in vitro water content and shown that the estimated in vivo water content from a measured susceptance value is approximately the same when a linear water concentration profile is assumed as when a non-linear profile is used for the basis of our calculations. This is at least correct if the parameters of the profile have physically realistic values. In this paper we have found relative in vivo water content (gram water per gram skin) and not absolute content. In order to find absolute water content of the S.C. from electrical susceptance measurements, the exact shape of the profile would be needed as well as the S.C. thickness of the area being considered. This should be a topic for future studies and would include more experimental methods in order to reveal the parameters in our model. If either of the parameters in the gradient are fixed, it would be a step towards an analytical expression of the absolute S.C. water content. The value of $a$, the water content of the S.C. surface, will depend on the relative humidity and temperature of the ambient air and will probably vary with time. Thus, the most likely candidate to be fixed is the water content, $d$, at the boundary of the S.C. towards viable skin. Viable skin contains a more or less stable content of water, independent of ambient variations such as relative hydration and temperature, which opens the possibility of boundary conditions to the innermost parts of the S.C.

An important question in this context is whether the interface towards the epidermis provides natural boundary conditions to the value of the innermost water content in the S.C. This should be a topic for future studies since literature seems ambiguous at this point. Occluding the S.C. while measuring susceptance may result in an asymptotic behavior of the susceptance due to a stable content of water in the S.C. corresponding to the level of hydration of the viable skin. Another possible method to search for the value of $d$ is from trans-epidermal-water-loss (TEWL)- measurements. Adjusting the relative humidity of the ambient air until TEWL vanishes probably means that the S.C. is hydrated at a level equal to $d$ throughout its entire thickness. The S.C. thickness varies due to swelling and that the number of keratin layers vary at different parts of the human body $[19,30]$. This is a problem yet to be solved. However, the interpersonal differences with respect to the S.C. thickness at equivalent parts of the body may be expected to be smaller, and therefore possible tabulated values of S.C. thickness with respect to location, age, sex etc. may show a better uniformity and possibly be good enough for our purpose.

It is also worth noticing that the in vitro water content is in general not the average of the in vivo water concentration profiles. This is as expected due to the capacitive properties of the S.C. where the outermost and driest parts of the S.C. contribute more to the impedance, and hence more to the estimated corresponding in vitro water content. This is, however, also a weakness in our model since then the estimated in vivo water content may not vary much with the changes in the shape of the innermost part of the water concentration profile.

\section{REFERENCES}

[1] I.H. Blank, "Factors which influence the water content of the stratum corneum", J. Invest. Dermatol., vol. 18, pp. 433-440, 1952.

[2] I.H. Blank, "Further observations on factors which influence the water content of the stratum corneum", J. Invest. Dermatol., vol. 21, pp. 259-271, 1953.

[3] R.J. Scheuplein, and I.H. Blank, "Permeability of the skin", Physiol. Rev., vol. 51, pp. 702-747, 1971.

[4] C. Blichmann, and J. Serup, "Hydration studies on scaly hand eczema", Contact Dermatitis, vol. 16, pp. 155-159, 1987.

[5] J. Fluhr, P. Elsner, E. Berardesca, and H.I. Maibach, Bioengineering of the Skin: Water and the Stratum Corneum, London: CRC Press, 2005, pp. 97-102.

[6] E.A. Holm, H.C. Wulf, L. Thomassen, and G.B.E. Jemec, "Instrumenatal assessment of atopic eczema: Validation of transepidermal water loss, stratum corneum hydration, erythema, scaling, and edema", J. Am. Acad. Dermatol., vol. 55, pp. 772-780, 2006.

[7] Ø.G. Martinsen, S. Grimnes, T. Kirkhus, L. Mørkrid, and K. Aas, "Increased moisture content in children's atopic skin", Eur. J. Pediatr. Dermatol., vol. 17, pp. 17-20, 2007.

[8] W.Y. Jang, J.J. Park, H.J. Jeong, H.S. Kim, and J.C. Park, "Time series trend of the water content on the different human anatomical sites using single frequency-susceptance measuring method", Proc. IEEE Int. Conf. (New York), 2006, pp. 3744-3746. 
[9] Ø.G. Martinsen, and S. Grimnes, "Facts and myths about electrical measurement of stratum corneum hydration state", Dermatology, vol. 202, pp. 87-89, 2001.

[10] Ø.G. Martinsen, S. Grimnes, and J. Karlsen, "Electrical methods for skin moisture assessment", Skin Pharmacology, vol. 8, pp. 237 $245,1995$.

[11] Ø.G. Martinsen, S. Grimnes, and O. Sveen, "Dielectric properties of some keratinized tissues. Part 1: Stratum corneum and nail in situ”, Med. Biol. Eng. Comput., vol. 35, pp. 172-176, 1997.

[12] Ø.G. Martinsen, S. Grimnes, J.K. Nilsen, C. Tronstad, W. Jang, H. Kim, and K. Shin, "Gravimetric method for in vitro calibration of skin hydration measurements", IEEE Trans. Biomed. Eng., vol. 55(2), pp. 728-732, 2008

[13] S. Grimnes, and Ø.G. Martinsen, "Sources of error in tetrapolar impedance measurements on biomaterials and other ionic conductors", J. Phys. D, vol. 40, pp. 9-14, 2007.

[14] S. Grimnes, "Impedance measurement of individual skin surface electrodes", Med. Biol. Eng. Comput., vol. 21, pp. 750-755, 1983.

[15] G.K. Johnsen, Ø.G. Martinsen, and S. Grimnes, "Water gradient and calibration of stratum corneum hydration measurements", Proc. IFMBE 2007, vol 17, pp. 158-160.

[16] R.R. Warner, M.C. Myers, and D.A. Taylor, "Electron probe analysis of human skin: determination of the water concentration profile", J. Invest. Dermatol., vol. 90, pp. 218-224, 1988.

[17] L. Chrit, P. Bastien, G.D. Sockalingum, D. Batisse, F. Leroy, M. Manfait, and C. Hadjur, "An in vivo randomized study of human skin moisturization by a new confocal raman fiber-optic microprobe: assessment of a glycerol-based hydration cream", Skin Pharmacol. Physiol., vol. 19, pp. 207-215, 2006.

[18] P.J. Caspers, G.W. Lucassen, E.A. Carter, H.A. Bruining, and G.J. Puppels, "In vivo confocal raman microspectroscopy of the skin: noninvasive determination of molecular concentration profiles", $J$. Raman Spectrosc., vol 31, pp. 813-818, 2001.

[19] T. Egawa, M. Hirao, and M. Takahashi, "In vivo estimation of stratum corneum thickness from water concentration profiles obtained with raman spectroscopy", Acta Derm. Venereol., vol. 87, pp. 4-8, 2007.

[20] I.H. Blank, J. Moloney, A.G. Emslie, I. Simon, and A. Charles, "The diffusion of water across the stratum corneum as a function of its water content", J. Invest. Dermatol., vol. 82, pp. 188-194, 1984.

[21] A. Fick, "Uber diffusion", Poggendorf's Ann. Phys., vol. 94, pp. 59-86, 1855.

[22] J. Crank, The Mathematics of Diffusion, England: Oxford University Press, 1956, pp. 258-261.

[23] M.S. Wu, "Water diffusivity and water concentration profile in human stratum corneum from transepidermal water los measurements", J. Soc. Cosmet. Chem., vol. 34, pp. 191-196, 1982.

[24] W. Montagna, and P.F. Parakkal, Eds., The Structure and Function of Skin, 3rd ed., London: Academic Press, 1974.

[25] R. Pethig, Dielectric and Electronic Properties of Biological Materials, New York: Wiley, 1997.

[26] Ø.G. Martinsen, S. Grimnes, and H.P. Schwan, "Interface phenomena and dielectric properties of biological tissue", Encycl. Surf. Coll. Sci., pp. 2643-2652, 2002.

[27] T. Yamamoto and Y. Yamamoto, "Electrical properties of the epidermal stratum corneum", Med. Biol. Eng., vol. 14, pp. 592-594, 1976.

[28] T. Yamamoto, and Y. Yamamoto, "Non-linear electrical properties of the skin in the low frequency range", Med. Biol. Eng. Comp., vol. 19, pp. 302-310, 1981.

[29] R. J. Scheuplein, "Molecular structure and diffusional processes across intact epidermis", Contract No. DA 18-108-AMC-148(A) Medical Research Laboratory, Edgewood Arsenal, Maryland, 1967.

[30] K.A. Holbrook and G.F. Odland, "Regional differences in the thickness (cell layers) of the human stratum corneum: an ultrastructural analysis", J. Invest. Dermatol., vol. 62, pp. 415-422, 1974.

(C) Johnsen et al.; Licensee Bentham Open.

This is an open access article licensed under the terms of the Creative Commons Attribution Non-Commercial License (http://creativecommons.org/licenses/by-nc/3.0/) which permits unrestricted, non-commercial use, distribution and reproduction in any medium, provided the work is properly cited. 